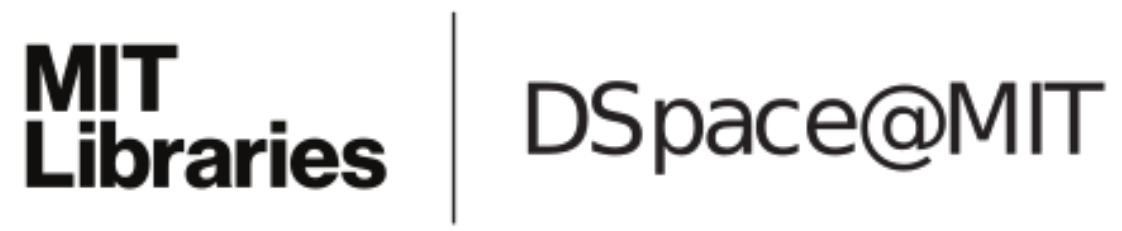

\author{
MIT Open Access Articles
}

A low-loss inductor structure and design
guidelines for high-frequency applications

The MIT Faculty has made this article openly available. Please share how this access benefits you. Your story matters.

Citation: Yang, Rachel S. et al. "A low-loss inductor structure and design guidelines for highfrequency applications." 2018 IEEE Applied Power Electronics Conference and Exposition (APEC), March 2018, San Antonio, Texas, USA, Institute of Electrical and Electronics Engineers (IEEE), April 2018 @ 2018 IEEE

As Published: http://dx.doi.org/10.1109/apec.2018.8341070

Publisher: Institute of Electrical and Electronics Engineers (IEEE)

Persistent URL: https://hdl.handle.net/1721.1/123505

Version: Author's final manuscript: final author's manuscript post peer review, without publisher's formatting or copy editing

Terms of use: Creative Commons Attribution-Noncommercial-Share Alike 


\section{A Low-Loss Inductor Structure and Design Guidelines for High-Frequency Applications}

\author{
Rachel S. Yang, Alex J. Hanson, David J. Perreault \\ Massachusetts Institute of Technology \\ 77 Massachusetts Avenue, Cambridge, MA 02139, USA \\ Email: rsyang@mit.edu, ajhanson@mit.edu, djperrea@mit.edu
}

\author{
Charles R. Sullivan \\ Thayer School of Engineering at Dartmouth \\ 14 Engineering Drive, Hanover, NH 03755, USA \\ Email: charles.r.sullivan@dartmouth.edu
}

\begin{abstract}
Operation in the $\mathrm{HF}$ regime (3-30 MHz) has shown potential for miniaturizing power electronics, but substantial challenges in the design of efficient miniaturized inductors at HF remain. At these frequencies, losses due to skin and proximity effects are difficult to reduce, and gaps needed to keep $B$ fields low in the core add fringing field loss. We propose a low-loss inductor structure suitable for small, highly efficient inductors at $\mathrm{HF}$ and introduce step-by-step design guidelines for the geometry. An example $\sim 15 \mu \mathrm{H}$ inductor designed using these guidelines achieved an experimental quality factor of 620 at $3 \mathrm{MHz}$ and $2 \mathrm{~A}$ (peak) of ac current. We further demonstrate the low loss of the inductor in a high-current-swing power converter operated at $1-3 \mathrm{MHz}$; at $250 \mathrm{~W}$, the inductor improved converter efficiency by $1.2 \%$, compared to a conventional inductor design. Thus, we show that the proposed inductor geometry and design guidelines can reduce losses and thereby help realize high frequency miniaturization of power electronics.
\end{abstract}

\section{INTRODUCTION}

Miniaturization of power electronics is often limited by the magnetic components due to high losses [1]. Although it has been shown that miniaturization is still available with increased frequencies into the $\mathrm{HF}(3-30 \mathrm{MHz})$ range [2], significant design challenges remain. Skin and proximity effects play large roles at HF, where conventional litz wire solutions become less practical due to manufacturing difficulties for strands thinner than a skin depth [3]. Therefore, other approaches for reducing proximity effect, such as single-layer windings or multi-layer foil windings, have been investigated [3]-[6]. Fringing fields from gaps in the core also significantly increase winding loss, and various winding configurations and materials have been explored to deal with these effects [3], [7], [8]. In particular, distributed or quasi-distributed gaps have successfully mitigated fringing field effects [9] and are beginning to be implemented in cores on the market [10].

Much research has also focused on modeling, ranging from finite element analysis (FEA) in-the-loop optimization [11] to analytical models of conductor loss [12]-[17] and core loss [18], [19], with some work targeting the HF range [20]. Although modeling can provide valuable analysis tools, it leaves unclear how to effectively design HF magnetic components. In addition, iterative optimization approaches can be slow and require a structure chosen a priori.

We propose an inductor structure suitable for highfrequency operation with large ac currents, along with analytic design guidelines that define the structure to maximize its quality factor. The proposed structure achieves high $Q$ through double-sided conduction in the winding and through quasi-distributed gaps. Section II provides an overview of the proposed inductor geometry. The design guidelines are discussed in Section III, and automation of the design process is outlined in Section IV. In Section V, an example design is provided for a $16.6 \mu \mathrm{H}$ inductor designed for $2 \mathrm{~A}$ (peak) of ac current at $3 \mathrm{MHz}$. The example achieves a simulated quality factor of 723 in FEA, and simulation results verify successful implementation of the design guidelines. In Section VI, we present a hardware prototype that achieves an experimental $Q$ of 620 , and describe details on measuring high quality factor at high frequency as well as possible sources of discrepancy. We also demonstrate the prototype inductor achieving improved efficiency and thermal results in a high-frequency, high-current-swing power converter $(1-3 \mathrm{MHz})$. We conclude that the proposed structure can achieve high $Q$ and that the analytic design guidelines are effective in designing high- $Q$ inductors operating at high frequency with large ac current components.

\section{GeOMetry OVERVIEW}

The proposed core geometry resembles a pot core, but has a particularly selected geometry with quasi-distributed gaps in the center post and outer shell (Fig. 1). To implement the
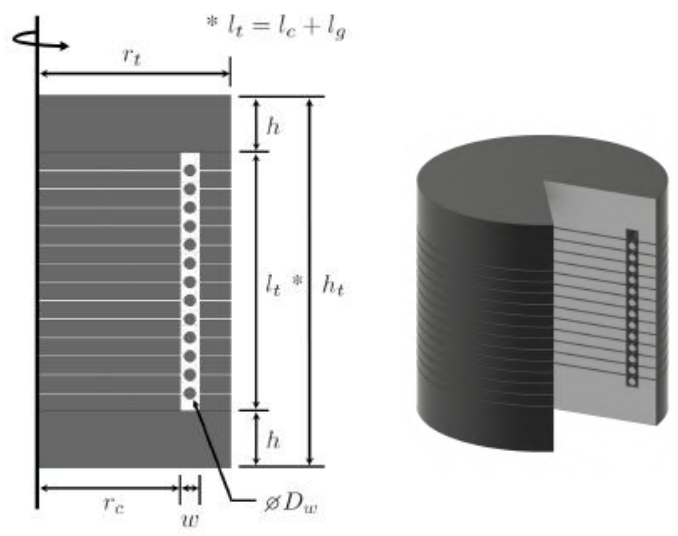

Fig. 1: Radial cross-sectional view (left) of the proposed inductor, with a center post, outer shell, and end caps encasing a single-layer winding. Parameters defining the geometry are labelled on this view. Revolving the cross-section about the axis of rotation produces the $3 \mathrm{D}$ model of the inductor on the right (a piece is cut out for clarity). 
quasi-distributed gaps, the core is composed of thin magnetically permeable discs and shell sections separated by small gaps. Magnetic end caps at the top and bottom of the structure bridge the center post and outer shell. A single-layer winding is centered in the window, with evenly spaced turns.

This structure uses a single-layer winding to reduce proximity effect losses and has a permeable return path to contain the flux and improve the predictability of the inductance. The quasi-distributed gaps help prevent fringing field losses while still allowing the use of a high-permeability core material. Properly designed, the structure can also utilize a large fraction of the winding cross-sectional area, as explained in Section III-B.

\section{DESIGN GUIDELINES}

The design guidelines below optimize the $Q$ of the proposed structure for a given volume and inductance. Most of the guidelines can be mathematically defined so that initial designs can be largely automated. A few of the parameters, however, must be manually tuned using the guidelines, as would be done in a non-analytic design process.

\section{A. Use quasi-distributed gaps to reduce gap fringing loss}

Gapping cores in high-current-swing applications is important for keeping $B$ fields low to reduce core loss, which scales as $B^{\beta}(\beta \approx 2-3)$, per the Steinmetz equation $P_{v}=C_{m} f^{\alpha} B^{\beta}$. However, as the frequency increases, the impact of fringing fields from the gaps on copper losses becomes more severe. To reduce the fringing loss, the proposed inductor uses quasidistributed gaps [9] as opposed to a conventional single lumped gap. Instead of dropping the entire MMF across one gap, the quasi-distributed gap has a smaller MMF across each of multiple gaps, causing less total loss in the winding. As shown in [9], the ratio of the pitch between the gaps $(p)$ to the spacing between the gaps and the conductor $(s)$ is an important parameter for fringing loss; [9] recommends $p<4 s$. While increasing the number of gaps at lower pitch reduces fringing loss, doing so also makes construction increasingly difficult. For the proposed structure, we set the number of gaps equal to the number of turns $\left(n_{g}=N\right)$; Appendix A discusses how this selection, in tandem with the guidelines in Sections III-D and III-E, meets the $p<4 s$ criterion of [9].

\section{B. Balance $H$ fields to achieve multi-sided conduction}

For a single-layer winding, copper loss at high frequencies is primarily due to skin effect, which reduces the effective area of current flow. In most cases, only a single side of the wire has a skin depth of conduction (not the entire circumference, as is commonly shown in textbooks for a wire in isolation). This single-sided conduction occurs because the $H$ fields near each turn in typical inductor geometries are imbalanced, causing uneven current distribution (Fig. 2a). If the $H$ fields on either side of the turn are balanced, double-sided conduction can be achieved to reduce copper loss (Fig. 2b).

\footnotetext{
${ }^{1}$ In a rod core inductor, due to fringing fields near the ends of the rod the single- and double-sided conduction argument only applies to the middle turns, so the images used here only capture those turns.
}

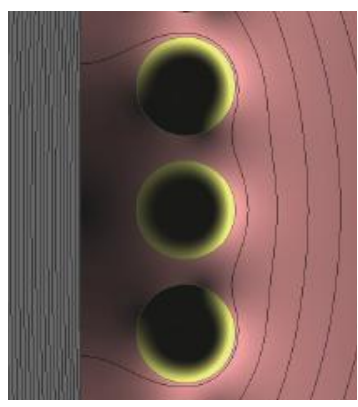

(a) Imbalanced $H$ fields

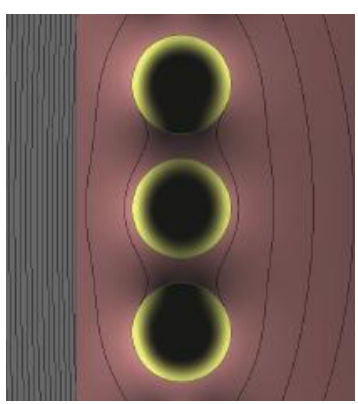

(b) Balanced $H$ fields
Fig. 2: When $H$ fields (red) are balanced, the effective conduction area in the winding (yellow) is increased. For example, a winding in a high permeability rod core inductor (2a), which has a lower $H$ field on the side of the winding near the core (grey) than away from it, has only single-sided conduction. A low permeability rod core inductor (2b) has comparable $H$ fields on either side of the winding, so the winding has double-sided conduction. The field balance/imbalance can also be seen in the plotted $B$ field lines. ${ }^{1}$

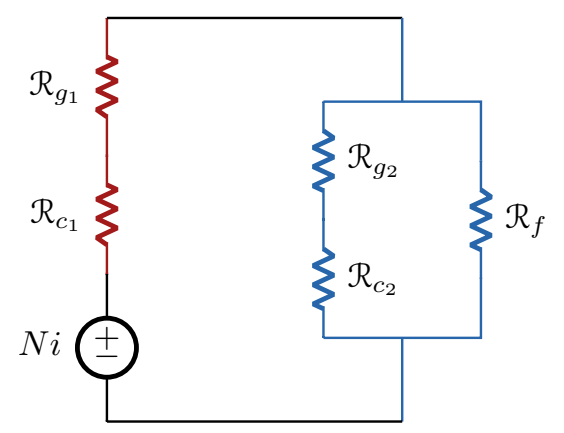

Fig. 3: Magnetic circuit model used to balance the $H$ fields in the proposed structure by making the reluctances of the center post (red) and the return path (blue) equal. This model includes the overall fringing field outside the structure but not the gap fringing fields. The discs of core material and the quasi-distributed gaps in the center post and the outer shell are treated as lumped reluctances. The end cap reluctances are assumed to be negligible.

To balance the $H$ fields, the center post and the return path need to have equal reluctances; doing so makes the MMF drop across the center post and the return path, and thus the $H$ field, the same (Fig. 3). To do this accurately, we include the overall fringing field outside the structure in the return path. Mathematically, we need

$$
\mathcal{R}_{c_{1}}+\mathcal{R}_{g_{1}}=\left(\mathcal{R}_{c_{2}}+\mathcal{R}_{g_{2}}\right) \| \mathcal{R}_{f}
$$

where $\mathcal{R}_{c_{1}}$ and $\mathcal{R}_{c_{2}}$ are, respectively, the lumped reluctance of the discs of core material in the center post and in the outer shell, $\mathcal{R}_{g_{1}}$ and $\mathcal{R}_{g_{2}}$ are, respectively, the lumped reluctance of the quasi-distributed gaps in the center post and in the outer shell, and $\mathcal{R}_{f}$ is the reluctance of the overall fringing path outside of the structure. 
$\mathcal{R}_{c_{1}}, \mathcal{R}_{c_{2}}, \mathcal{R}_{g_{1}}$, and $\mathcal{R}_{g_{2}}$ can be calculated directly from the geometry (Fig. 1):

$$
\begin{aligned}
\mathcal{R}_{c_{1}} & =\frac{l_{c}}{\mu_{c} \pi r_{c}^{2}} \quad \text { (2) } \quad \mathcal{R}_{c_{2}}=\frac{l_{c}}{\mu_{c} \pi\left(r_{t}^{2}-\left(r_{c}+w\right)^{2}\right)} \\
\mathcal{R}_{g_{1}} & =\frac{l_{g}}{\mu_{0} \pi r_{c}{ }^{2}} \quad \text { (4) } \quad \mathcal{R}_{g_{2}}=\frac{l_{g}}{\mu_{0} \pi\left(r_{t}^{2}-\left(r_{c}+w\right)^{2}\right)}
\end{aligned}
$$

where $l_{c}$ is the overall height of the core material discs, $l_{g}$ is the overall length of the gap, and $\mu_{c}$ is the permeability of the core material.

$\mathcal{R}_{f}$, however, is more difficult to estimate. Since the proposed inductor and a solenoid have similar overall fringing fields, we can back out the fringing field reluctance $\mathcal{R}_{f}$ of the proposed inductor from any appropriate solenoid inductance model. In general, for a solenoid,

$$
L=\frac{N^{2}}{\mathcal{R}_{\text {inside }}+\mathcal{R}_{f}}
$$

where $\mathcal{R}_{\text {inside }}$ is the reluctance of the path through the center of the solenoid. By substituting (6) into a solenoid inductance model of our choosing, we can then derive an expression for $\mathcal{R}_{f}$ to use for balancing the $\mathrm{H}$ fields of the proposed inductor. For structures where $h_{t}>\frac{2}{3} r_{t}$, the following SI air-core solenoid model [21] can be used:

$$
L \approx \frac{\mu_{0} N^{2} \pi r_{t}^{2}}{h_{t}+0.9 r_{t}}
$$

We can then back out

$$
\mathcal{R}_{f} \approx \frac{0.9}{\mu_{0} \pi r_{t}}
$$

For more general cases, the short solenoid model [22] may be more appropriate:

$$
\widetilde{L} \approx 2 F N^{2} \widetilde{r_{t}}
$$

where $\widetilde{L}$ is in microhenries and $\widetilde{r_{t}}$ is in inches. $F$ is an experimentally derived quantity, as defined in [22]. Using this model, $\mathcal{R}_{f}$ would be

$$
\mathcal{R}_{f} \approx \frac{2.54 \times 10^{4}}{2 r_{t} F}-\frac{h_{t}}{\mu_{0} \pi r_{t}^{2}}
$$

Using $\mathcal{R}_{f}$, we can then design the center post and the return path to have equal reluctances, and thus balance the $H$ fields to achieve double-sided conduction.

\section{Distribute B fields to reduce overall core loss}

While $H$ field balancing helps prevent circulating current losses in the winding, evenly distributed $B$ fields in the core can reduce core loss. In the case of unevenly distributed $B$ fields, since core loss scales as $B^{\beta}$, regions with higher $B$ fields experience much greater core loss, leading to a greater total core loss. Since the center post and the shell of the proposed inductor are designed to have the same effective permeability, a design which achieves balanced $H$ fields near the winding will also achieve evenly distributed $B$ fields in these core regions.

For cases in which the center post and the outer shell do not have the same effective permeability, the structure cannot achieve both balanced $H$ fields and evenly distributed $B$ fields. Instead, to minimize overall loss, the designer would need to find the optimal balance with partial double-sided conduction and a slight imbalance in the $B$ field distribution.

For the end caps, the $B$ field distribution, and thus core loss, is affected by their thickness. Thicker end caps allow the $B$ field to distribute more in these regions for lower core loss but increase the volume of the inductor, with diminishing returns for large end cap thickness. The designer can use FEA to determine an end cap thickness to reduce loss without excessive volume.

\section{Select a wire size that optimizes effective conduction area}

Since the structure is designed to achieve multi-sided conduction in the winding, larger diameter wire reduces copper loss by providing more circumferential conduction area. As the wire diameter increases, however, proximity effect losses between the turns begin to play a larger role. Conduction in the top and bottom of each turn is then lost, reducing the effective conduction area and thus increasing copper loss.

Simulations show that selecting a wire diameter such that the vertical fill of the winding in the window is $50-80 \%$ optimizes the total effective conduction area for these two competing effects (Fig. 4). For a given window height, the copper loss is largely insensitive to changes in the wire diameter near the optimum.

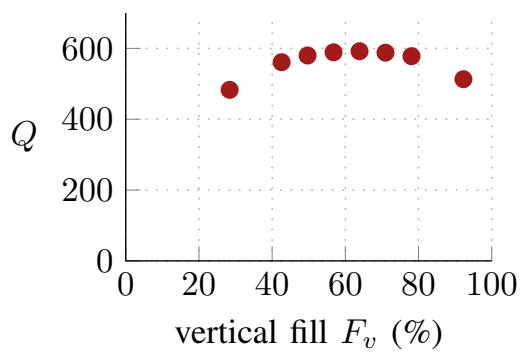

Fig. 4: For a given window height, a wire diameter that yields a 50-80\% vertical window fill optimizes the total effective conduction area to reduce copper loss. To find this optimum, inductors with the same inductance and core geometry but different winding diameters were simulated. All inductors had a large window width to make the gap fringing loss on the winding negligible.

\section{E. Select a window size that balances gap fringing field loss and core loss in end caps to reduce overall loss}

To minimize gap fringing field loss, the structure would ideally have a large window to increase the horizontal distance between the gaps and the winding. However, since flux crowding around the ends of the window leads to higher $B$ fields in and near the end caps (Fig. 5), a larger window would increase core loss by increasing the volume of these high- $B$ field regions. 


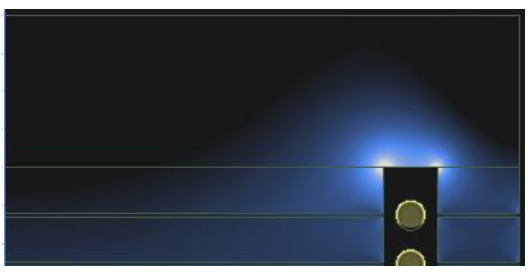

Fig. 5: Flux crowding at the end of the window leads to higher $B$ fields (white and light blue) and thus greater core loss.

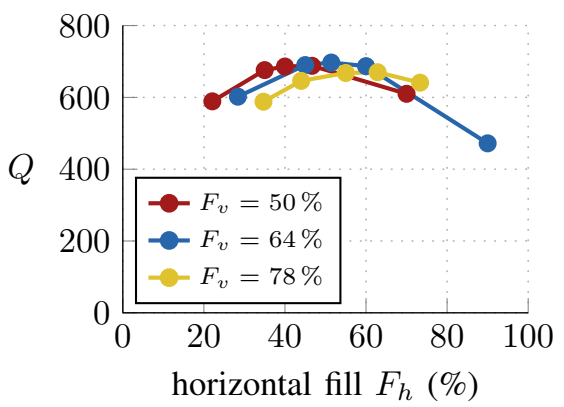

Fig. 6: For a given wire diameter, a window size with a $40-60 \%$ horizontal fill for the winding balances the gap fringing loss and end cap core loss. To find this balance, inductors with the same inductance and volume but different window widths were simulated. As shown in the graph, the optimal range of horizontal fill holds across the optimal vertical fill $\left(F_{v}\right)$ range.

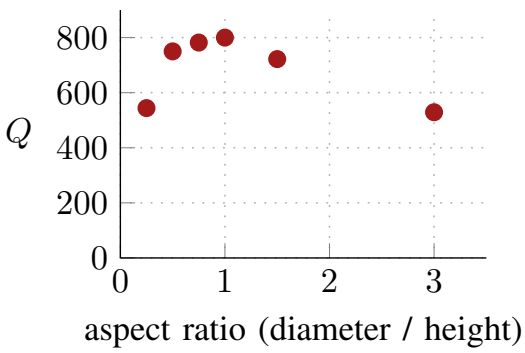

Fig. 7: Structures with a "square" aspect ratio achieve the optimum $Q$. To find this optimum, inductors with different aspect ratios but the same inductance and volume were simulated, and each design was optimized using the guidelines discussed in Sections III-A to III-F.

Simulations show that to balance the fringing loss and the end cap core loss, the horizontal fill of the winding in the window should be between 40-60\% (Fig. 6). In other words, for a given wire diameter $D_{w}$, the optimal window size is approximately $2 D_{w}$, but the overall loss is largely insensitive to changes in the window size near the optimum.

\section{F. Balance copper and core loss to reduce overall loss}

As in conventional inductor designs, for a given core material, the number of turns and overall gap length can be used to tune the copper and core losses. The overall loss is usually minimized at a point where core loss is close to, but slightly less than winding loss [23]. To achieve this, the designer can model the losses with exact core loss parameters or hand-tune in FEA.

\section{G. Use a square aspect ratio to minimize overall loss}

A "square" aspect ratio (diameter $\approx$ height) is the preferred overall geometry for this structure. FEA simulations of otherwise optimized inductors show that structures that are much wider than they are tall, or vice-versa, achieve lower $Q$ (Fig. 7). Wide and flat structures have fewer turns and less granularity to balance core and copper loss, while tall and thin structures have more core material and correspondingly greater core loss.

\section{Automating Initial Designs of THE PROPOSED INDUCTOR STRUCTURE}

Using the design guidelines discussed in Section III, we can mathematically define the proposed inductor geometry. The design process can then be largely automated to generate high$Q$ inductor designs for a desired volume and inductance at a given frequency and current (Fig. 8). The end cap height and the number of turns, however, must still be manually tuned.

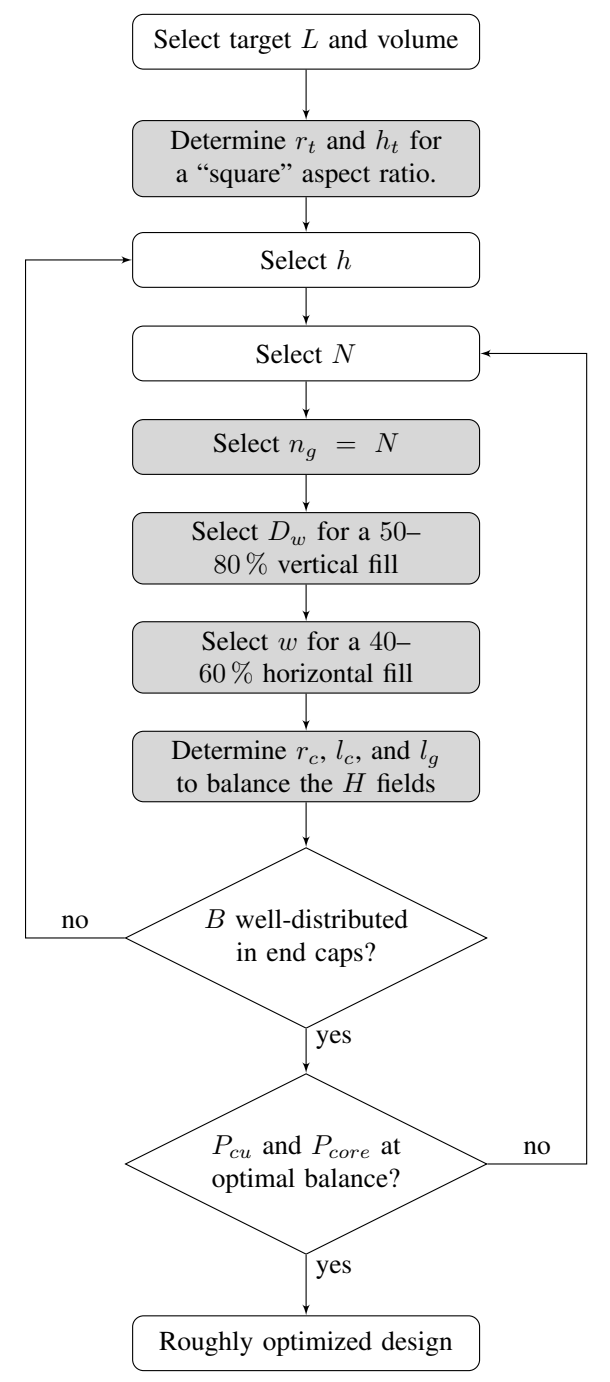

Fig. 8: Flowchart of the design process for the proposed structure using the guidelines presented in Section III. The parameters used in the flowchart are labelled on the cross-sectional view in Fig. 1. Grey fill denotes steps that can be automated. 


\section{An EXAmple $16.6 \mu \mathrm{H}$ Design - Simulations}

Using the design guidelines in Section III, we simulated an example $16.6 \mu \mathrm{H}$ inductor that achieved a simulated $Q$ of 723 (Table I). To design the example inductor, the target inductance and volume as well as a selected $h$ and $N$ were entered into a script that generated dimensions for the geometry, which was then simulated. Then, the height of the end caps was manually tuned so that the $B$ fields were well-distributed, and the script was re-run with the optimized $h$. Next, designs with varying number of turns were generated using the script to find the optimum core and copper loss balance. At this point, the example design was roughly optimized, but we chose to continue with additional minor tweaking in FEA for further optimization.

TABLE I: Specifications for the simulated example inductor

\begin{tabular}{ll}
\hline \hline Inductance & $16.6 \mu \mathrm{H}$ \\
\hline Frequency & $3 \mathrm{MHz}$ \\
\hline Current & $2 \mathrm{~A}($ peak, ac) \\
\hline Diameter & $26.9 \mathrm{~mm}$ \\
\hline Height & $26.0 \mathrm{~mm}$ \\
\hline Core Material & Fair-Rite $67, \mu_{r}=40$ \\
& $C_{m}=1474.5 \mathrm{~W} / \mathrm{cm}^{3}$, \\
& $\alpha=1.25, \beta=3.29$ \\
\hline Wire Gauge & $20 \mathrm{AWG}$ \\
\hline \hline
\end{tabular}

The simulation results verified that the example design followed all of the guidelines for achieving a roughly optimized $Q$. The $B$ fields were roughly evenly distributed between the center post and the shell for low core loss (Fig. 9a), and most of the turns had balanced $H$ fields and associated double-sided conduction for low copper loss (Fig. 9b). It was verified that additional thickness to the end caps would have minimal effect on loss, and that larger or smaller window sizes would increase total loss. The core loss and copper loss were also verified to be optimally balanced.

\section{AN EXAMPLE 16.6 $\mu$ H DESIGN: EXPERIMENTAL RESULTS}

A prototype (Fig. 10) of the example inductor presented in Section V was constructed; fabrication details are shown in Appendix B. The prototype inductor achieved a largesignal quality factor measurement of $Q=620$ at $3 \mathrm{MHz}$ and $2 \mathrm{~A}$ (peak) of ac current (Table II). Below, we discuss our approach for measuring high $Q$ at high frequency. We also show the prototype improving the efficiency and thermal results of a high-current-swing power converter. Finally, we discuss possible sources of discrepancy between the simulated and experimental quality factors.

TABLE II: The simulated example inductor and the prototype

\begin{tabular}{lcc}
\hline & Simulated & Prototype \\
\hline Inductance & $16.6 \mu \mathrm{H}$ & $13.5 \mu \mathrm{H}$ \\
$Q$ at $3 \mathrm{MHz}, 2 \mathrm{~A}$ (peak, ac) & 723 & 620 \\
\hline
\end{tabular}

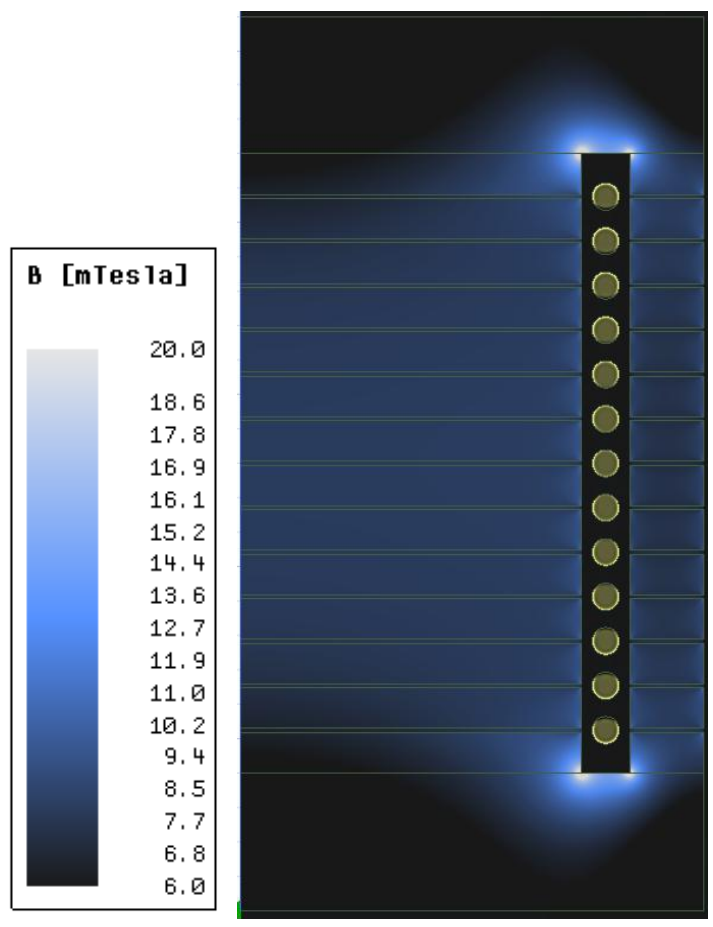

(a) Roughly even distribution of $B$ fields

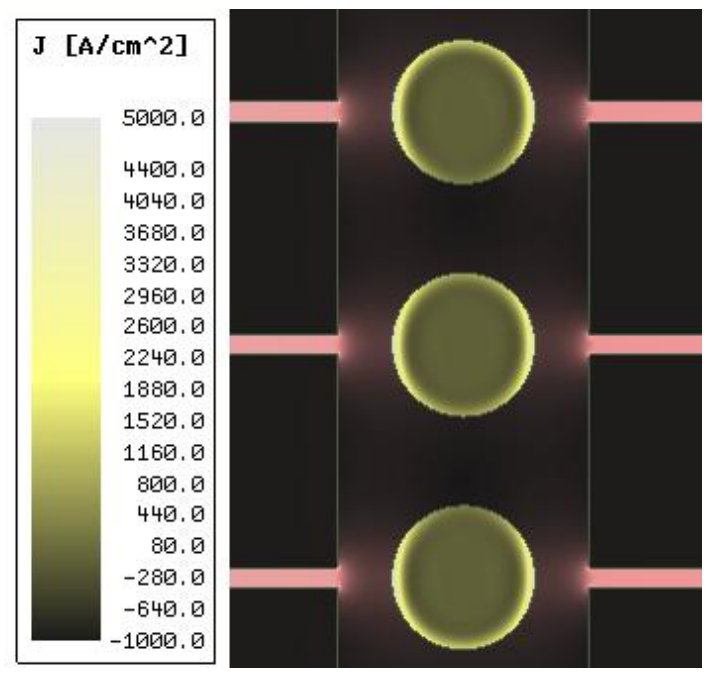

(b) Turns with multi-sided conduction

Fig. 9: $B$ field (blue), $H$ field (red), and current distribution (yellow) simulations of the example $16.6 \mu \mathrm{H}$ inductor verifying that it follows the design guidelines in Section III. These simulations are of the "worst-case" distributions for a helical winding, with each turn next to a gap. Other cross sections in a prototype would have turns in between the gaps with even lower loss.

\section{A. Measuring high $Q$ (large-signal) by minimizing probe loss and including capacitor ESR}

To measure the large-signal $Q$ of the prototype, we used the same resonant measurement approach from [2] and [24] with some modifications for measuring high $Q$. This approach operates a series LC circuit at resonance so that the ratio of the peak capacitor voltage to the peak input voltage can be 


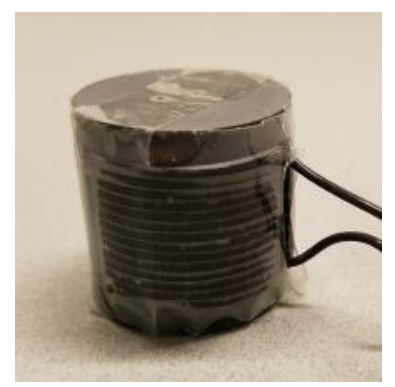

Fig. 10: Prototype inductor. Vertical windows in the outer shell were added to impede the circumferential component of flux and to allow the winding terminations to leave the structure.

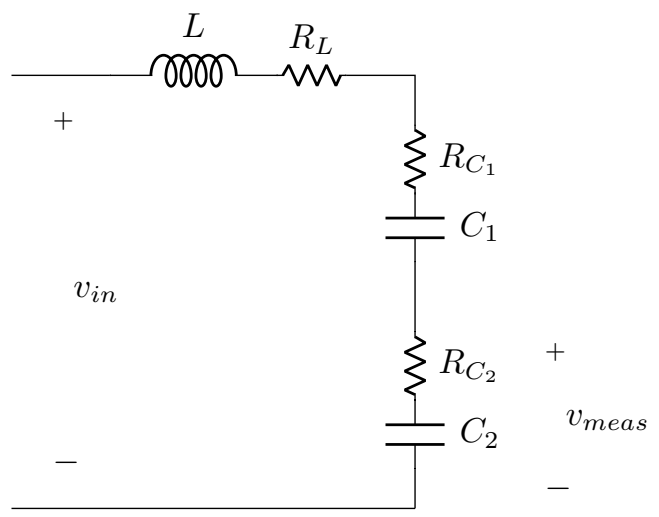

Fig. 11: Circuit using the resonant measurement approach to measure high $Q$, modified to include a capacitor divider to step down the measured output voltage. The ESRs of the capacitors are also included.

approximated as the $Q$ of the inductor. For a high $Q$ inductor, however, the probe loss when measuring the high-frequency, high-voltage output signal can significantly affect results. To get a more accurate measurement of the resonant capacitor voltage, we replaced the capacitor with a capacitor divider having the same net impedance. The stepped-down voltage was then measured with minimal probe loading (Fig. 11).

In addition, for measuring high $Q$, the approximation made in [2] and [24] that the equivalent series resistances (ESRs) of the capacitors $\left(R_{C_{1}}, R_{C_{2}}\right)$ are small compared to the equivalent series resistance of the inductor $\left(R_{L}\right)$ no longer holds, even with NP0, porcelain, or mica capacitors. Instead, we include the capacitor ESRs in deriving an expression for the quality factor of the inductor $\left(Q_{L}\right)$ using the measured input voltage $v_{i n}$ and stepped-down voltage $v_{\text {meas }}$.

From Fig. 11, we can see that at resonance, since the impedances of the inductor and capacitors cancel,

$$
\frac{V_{\text {meas }, p k}}{V_{\text {in }, p k}}=\left|\frac{R_{C_{2}}+\frac{1}{j \omega_{0} C_{2}}}{R_{C_{1}}+R_{C_{2}}+R_{L}}\right|
$$

We also know that at resonance,

$$
Q_{L}=\frac{\omega_{0} L}{R_{L}}
$$

From (11) and (12), the quality factor of the inductor as a function of $V_{i n, p k}$ and $V_{\text {meas,pk }}$ is

$$
Q_{L}=\frac{\omega_{0} L}{\frac{V_{\text {in, }, p k}}{V_{\text {meas }, p k}} \sqrt{{R_{C_{2}}{ }^{2}+\left(\frac{1}{\omega_{0} C_{2}}\right)^{2}}^{2}-R_{C_{1}}-R_{C_{2}}}}
$$

where $R_{C_{1}}$ and $R_{C_{2}}$ are the ESR values found on the datasheet for the capacitors.

In cases where the capacitors are physically composed of multiple capacitors in parallel, the ESRs $R_{C_{1}}$ and $R_{C_{2}}$ can each be approximated as the equivalent parallel resistance for the corresponding ESRs. $C_{2}$ can also be approximated as the equivalent parallel capacitance of the capacitors comprising it.

\section{B. Resonant measurement approach validated using an air-} core inductor

We validated the large-signal measurement approach described in Section VI-A with small-signal $Q$ measurements of an air-core inductor. Since an air-core inductor has no nonlinear core loss, its small-signal and large-signal quality factors should be the same. Based on the equivalent series resistance of an air-core inductor measured at $3 \mathrm{MHz}$ with an impedance analyzer, the small-signal quality factor of the inductor was calculated to be $Q=540$ at this frequency. Based on the resonant measurement approach, the same aircore inductor had a measured quality factor of $Q=500$ at $3 \mathrm{MHz}$ and $2 \mathrm{~A}$, which validates this approach for measuring large-signal $Q$ to within $10 \%$.

\section{Prototype inductor improved efficiency of a high-current- swing power converter}

In addition to achieving a high $Q$ of 620 under controlled conditions, the example inductor was used in a power-factorcorrection power converter operating at dynamically varying frequencies of 1-3 MHz [25]. The inductor improved converter performance significantly (Fig. 12) over a more conventional open-magnetic-circuit inductor, despite having similar effective volume. This improvement can also be seen in thermal measurements: at a $93 \mathrm{~W}$ operating point, the conventional inductor saw a $\sim 30^{\circ} \mathrm{C}$ temperature rise, while at a much higher power $(296 \mathrm{~W})$, the proposed inductor only saw a $\sim 3{ }^{\circ} \mathrm{C}$ rise (Fig. 13).

\section{Discrepancy in $Q$ may be explained by the prototype construction process}

The difference between the simulated and manufactured $Q$ of the example inductor ( $Q=723$ vs. $Q=620$ ) could be attributed to several sources. The three vertical windows in the outer shell, which were added to impede circumferential flux and to allow the winding terminations to leave the structure, would have introduced additional fringing loss on the sections of winding nearby. The prototype was also assembled by hand, so the core pieces in the structure were not in perfect alignment, which would affect field shaping.

One effect that may substantially reduce performance in some designs is increased losses on the surfaces of ferrite 


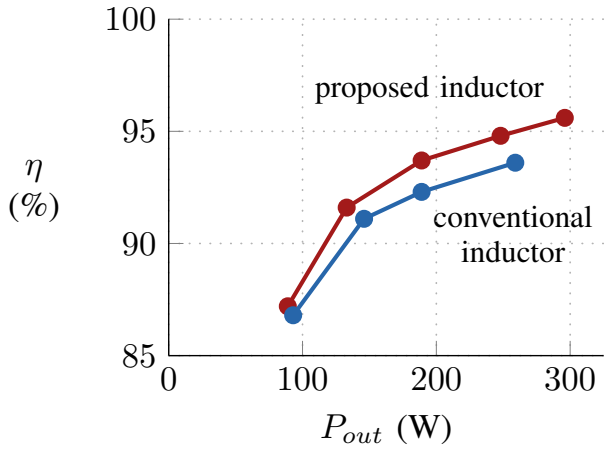

Fig. 12: Efficiencies of a power converter operating at $1-3 \mathrm{MHz}$ at different output powers using the proposed inductor versus a conventional inductor.

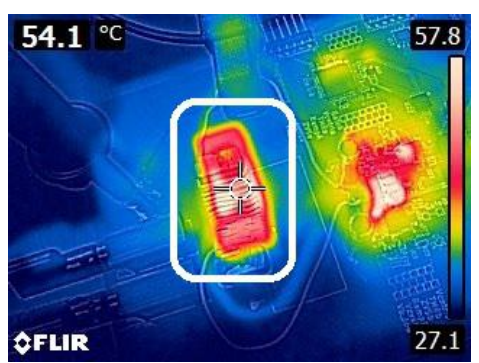

(a) conventional inductor $\left(\Delta T=\sim 30^{\circ} \mathrm{C}\right.$ at $\left.P_{\text {out }}=93 \mathrm{~W}\right)$

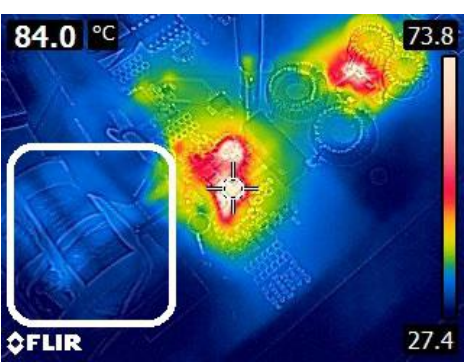

(b) proposed inductor $\left(\Delta T=\sim 3{ }^{\circ} \mathrm{C}\right.$ at $\left.P_{\text {out }}=296 \mathrm{~W}\right)$

Fig. 13: Thermal images showing the proposed inductor (13b, white box) having a much smaller temperature rise for a higher converter output power than a more conventional open-magnetic-circuit inductor (13a, white box).

parts. The multiple gaps increase the surface area and have been observed to greatly increase loss in some MnZn ferrite quasi-distributed designs [26]. The high $Q$ of our prototype indicates that if such effects are present in our core, they are relatively minor.

\section{CONCLUSION}

Design of highly efficient, miniaturized inductors in the $\mathrm{HF}$ range is a significant challenge. The proposed inductor structure and design approach provide a solution for lowloss high frequency power inductors. Using a set of analytic design guidelines, designers can achieve a roughly optimized inductor for a desired inductance and volume and then choose to further refine the design in FEA using the general design rules. This geometry and its guidelines for achieving high $Q$ were confirmed experimentally through an example inductor with a manufactured $Q$ of 620 .

\section{ACKNOWLEDGMENT}

This work was supported by the National Science Foundation under Grant 1609240 and by Analog Devices through the MIT SuperUROP program. We would also like to thank FairRite for manufacturing the magnetic core pieces used in the prototype.

\section{APPENDIX A}

\section{DESIGNING THE DISTRIBUTED GAP GEOMETRY TO MINIMIZE GAP FRINGING LOSS}

In this section, we show that setting the number of gaps $n_{g}$ equal to the number of turns $N$ aligns closely with the rule of thumb for minimizing gap fringing loss from [9], where the pitch between gaps $(p)$ should be less than four times the spacing between the gap and the conductor $(s)$, or $p<4 s$. We assume a large $N$ so that the center-to-center spacing between each turn can be approximated as $F_{v}$.

Mathematically, $F_{v}$ and $F_{h}$ are

$$
F_{v} \approx \frac{D_{w}}{D_{w}+v} \quad(14) \quad F_{h}=\frac{D_{w}}{w}
$$

where $v$ is the edge-to-edge vertical spacing between each turn and $D_{w}$ and $w$ are the parameters defined in Fig. 1. Based on the geometry, the spacing between the gap and the winding is

$$
s=\frac{w-D_{w}}{2}
$$

Since we set $n_{g}=N$, the pitch between gaps is

$$
p=D_{w}+v
$$

By combining (14), (15), (16), and (17), we get that

$$
\frac{p}{s}=\frac{2}{F_{v}} \frac{F_{h}}{1-F_{h}}
$$

Most combinations of $F_{v}$ and $F_{h}$ within the recommended ranges (Sections III-D and III-E) satisfy the design criteria from [9], $p<4 s$. For example, for values in the center of these ranges, $F_{v}=0.65$ and $F_{h}=0.50, p / s=3.1<4$. At the edge of these ranges where $F_{v}$ is small and $F_{h}$ is large, the $p / s$ ratio surpasses this rule of thumb, with the worst case at $p / s=6$, when $F_{v}=0.50$ and $F_{h}=0.60$. These edge cases, however, still achieve roughly optimal designs. Therefore, setting $n_{g}=N$ yields designs for the proposed inductor that meet (or nearly meet) the design criterion of [9] and thus, achieve roughly optimum $Q$.

\section{APPENDIX B PROTOTYPE CONSTRUCTION}

The prototype inductor was constructed modularly with the aid of custom 3D-printed fixtures. The center post was constructed first (Fig. 14a) with one of the end caps. To control the quasi-distributed gaps, we stacked laser cut pieces of shimstock with the appropriate thickness ( 4.5 mils) in between each layer of core material. To center all of the layers of the centerpost, a $1 \mathrm{~mm}$ diameter hole was drilled in the center of the discs and the center post shimstock pieces so they could be assembled on a rod. Since the drilled holes were relatively small, we expect minimal effect on the fields.

For the winding, $20 \mathrm{AWG}$ solid core wire with Teflon fluorinated ethylene propylene (FEP) insulation was wound around a 3D-printed fixture of the same diameter as the center 
post (Fig. 14b). The wire was chosen to have the appropriate insulation thickness (9 mils from the conductor diameter to the outer diameter) to center it in the window. Then, the winding was wrapped in a single layer of 3.1 mils thick polypropylene tape (package sealing tape) to maintain its shape, removed from the fixture and put on the center post.

The outer shell, composed of three sections to allow for vertical windows (with approximate widths of $1.5 \mathrm{~mm}$ ), was constructed one section at a time. Each section was stacked on a 3D-printed fixture, alternating between layers of core material and laser cut shimstock (Fig. 14c). The outer surface of each section was taped to hold all the pieces together. Then, the sections were added to the center post structure so that the two winding terminations could leave the structure through one of the vertical windows in the shell.

Afterwards, the second end cap was added, and the rod was removed from the centerpost. Finally, the entire circumference of the inductor was wrapped with a single layer of packing tape to apply radial pressure, and a strip of packing tape was wrapped vertically around the inductor to apply vertical pressure.

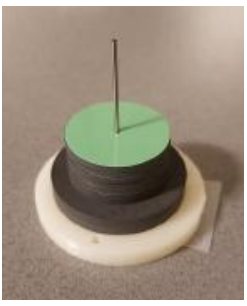

(a) Center post

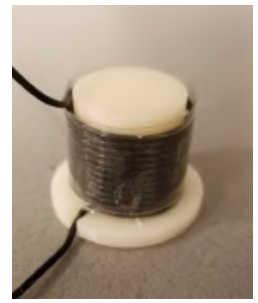

(b) Winding

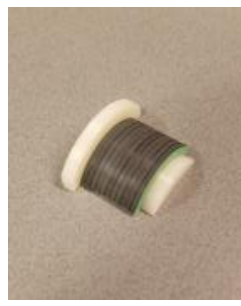

(c) Outer shell section
Fig. 14: Construction of the prototype inductor using custom 3Dprinted fixtures (white).

\section{REFERENCES}

[1] C. R. Sullivan, B. A. Reese, A. L. F. Stein, and P. A. Kyaw, "On size and magnetics: Why small efficient power inductors are rare," in 2016 International Symposium on 3D Power Electronics Integration and Manufacturing (3D-PEIM), June 2016, pp. 1-23.

[2] A. J. Hanson, J. A. Belk, S. Lim, C. R. Sullivan, and D. J. Perreault, "Measurements and performance factor comparisons of magnetic materials at high frequency," IEEE Transactions on Power Electronics, vol. 31, no. 11, pp. 7909-7925, Nov 2016.

[3] C. R. Sullivan, "Prospects for advances in power magnetics," in CIPS 2016; 9th International Conference on Integrated Power Electronics Systems, March 2016, pp. 1-9.

[4] P. A. Kyaw, A. L. F. Stein, and C. R. Sullivan, "High-q resonator with integrated capacitance for resonant power conversion," in 2017 IEEE Applied Power Electronics Conference and Exposition (APEC), March 2017, pp. 2519-2526.

[5] A. L. F. Stein, P. A. Kyaw, and C. R. Sullivan, "High-q self-resonant structure for wireless power transfer," in 2017 IEEE Applied Power Electronics Conference and Exposition (APEC), March 2017, pp. 3723 3729.

[6] C. R. Sullivan, "Aluminum windings and other strategies for highfrequency magnetics design in an era of high copper and energy costs," in APEC 07 - Twenty-Second Annual IEEE Applied Power Electronics Conference and Exposition, Feb 2007, pp. 78-84.

[7] J. Qiu, A. Hanson, and C. Sullivan, "Design of toroidal inductors with multiple parallel foil windings," in 14th Workshop on Control and Modeling for Power Electronics (COMPEL). IEEE, 2013, pp. 23-26.
[8] Y. Han and D. J. Perreault, "Inductor design methods with lowpermeability rf core materials," IEEE Transactions on Industry Applications, vol. 48, no. 5, pp. 1616-1627, Sept 2012.

[9] J. Hu and C. R. Sullivan, "The quasi-distributed gap technique for planar inductors: design guidelines," in Industry Applications Conference, 1997. Thirty-Second IAS Annual Meeting, IAS '97., Conference Record of the 1997 IEEE, vol. 2, Oct 1997, pp. 1147-1152 vol.2.

[10] "Distributed air gaps in ferrite cores," EPCOS AG - a TDK Group Company, Tech. Rep., June 2017. [Online]. Available: https: //de.tdk.eu/download/2113422/321697054fce0c768ea66959fde3b3db/ ferrites-air-gaps-pb.pdf

[11] Z. Zhang, K. D. T. Ngo, and J. L. Nilles, "Design of inductors with significant ac flux," IEEE Transactions on Power Electronics, vol. 32, no. 1, pp. 529-539, Jan 2017.

[12] P. L. Dowell, "Effects of eddy currents in transformer windings," Electrical Engineers, Proceedings of the Institution of, vol. 113, no. 8, pp. 1387-1394, August 1966

[13] C. R. Sullivan, "Optimal choice for number of strands in a litz-wire transformer winding," IEEE Transactions on Power Electronics, vol. 14, no. 2, pp. 283-291, Mar 1999.

[14] W. G. Hurley, E. Gath, and J. G. Breslin, "Optimizing the ac resistance of multilayer transformer windings with arbitrary current waveforms," IEEE Transactions on Power Electronics, vol. 15, no. 2, pp. 369-376, Mar 2000.

[15] T. Delaforge, H. Chazal, J. L. Schanen, and R. J. Pasterczyk, "Increasing windings efficiency at high frequencies: Hollow conductors and clad metal conductors formal solution based on the magnetic potential," in 2015 IEEE Energy Conversion Congress and Exposition (ECCE), Sept 2015, pp. 5689-5695.

[16] X. Nan and C. R. Sullivan, "An improved calculation of proximityeffect loss in high-frequency windings of round conductors," in Power Electronics Specialist Conference, 2003. PESC '03. 2003 IEEE 34th Annual, vol. 2, June 2003, pp. 853-860 vol.2.

[17] M. Chen, M. Araghchini, K. K. Afridi, J. H. Lang, C. R. Sullivan, and D. J. Perreault, "A systematic approach to modeling impedances and current distribution in planar magnetics," IEEE Transactions on Power Electronics, vol. 31, no. 1, pp. 560-580, Jan 2016.

[18] K. Venkatachalam, C. R. Sullivan, T. Abdallah, and H. Tacca, "Accurate prediction of ferrite core loss with nonsinusoidal waveforms using only steinmetz parameters," in 2002 IEEE Workshop on Computers in Power Electronics, 2002. Proceedings., June 2002, pp. 36-41.

[19] J. Muhlethaler, J. Biela, J. W. Kolar, and A. Ecklebe, "Improved coreloss calculation for magnetic components employed in power electronic systems," IEEE Transactions on Power Electronics, vol. 27, no. 2, pp. 964-973, Feb 2012.

[20] C. Fei, F. C. Lee, and Q. Li, "High-efficiency high-power-density llc converter with an integrated planar matrix transformer for high output current applications," IEEE Transactions on Industrial Electronics, vol. PP, no. 99, pp. 2428-2435, 2017.

[21] T. H. Lee, Planar Microwave Engineering: A Practical Guide to Theory, Measurement, and Circuits, 1st ed. Cambridge University Press, 2004, pp. $140-142$.

[22] F. E. Terman, Radio Engineers' Handbook, 1st ed. McGraw-Hill Book Company, Inc., 1943, pp. 52-55.

[23] V. Valchev and A. Van den Bossche, Inductors and Transformers for Power Electronics. CRC Press, 2005.

[24] Y. Han, G. Cheung, A. Li, C. Sullivan, and D. Perreault, "Evaluation of magnetic materials for very high frequency applications," IEEE Transactions on Power Electronics, pp. 425-435, 2008.

[25] A. J. Hanson and D. J. Perreault, "A high frequency power factor correction converter with soft switching," in IEEE Applied Power Electronics Conference (APEC), 2018.

[26] J. W. Kolar, F. Krismer, M. Leibl, D. Neumayr, L. Schrittwieser, and D. Bortis, Impact of Magnetics on Power Electronics Converter Performance - State-of-the-Art and Future Prospects, Keynote Presentation at the PSMA Workshop on "Power Magnetics @ High Frequency - Transforming the Black Magic to Engineering", Tampa, FL, USA, March 25, 2017. 